\title{
Sleeve Fracture of the Patella: A Case Report
}

\author{
Virinderpal Singh Chauhan, ${ }^{1}$ Michael Maru ${ }^{2}$
}

Rift Valley Provincial General Hospital, Nakuru

PCEA Kikuyu Hospital, Nairobi

Correspondence to: Dr. Virinderpal Chauhan, PO Box 3273-20100 Nakuru, Kenya; Email: dr.chauhansingh@gmail.com

\section{Summary}

Sleeve fractures form an integral part of the patellar fractures seen in children, which in their own entity are rare injuries. Diagnosis is usually missed early due to lack of awareness, and X-ray features are tricky if one is not aware of or familiar with the anatomy of an immature patella. This propagates neglect and outcomes are undesirable for the patient. We present a case of a 12-year-old boy who presented to our facility with a sleeve fracture that was initially missed in the outpatient clinic. Open reduction and repair with nonabsorbable sutures was done and the construct protected

\section{Introduction}

Sleeve fractures are rare in the skeletally immature child and represent chondral or osteochondral injuries that occur between the cartilage sleeve and the main part of the patella and ossific nucleus. Fractures of the patella collectively form $1 \%$ of all fractures, with sleeve fractures representing $57 \%$ of these (1-3). This is due to the anatomy of the immature patella, which begins to ossify at about 3-6 years of age and is surrounded by a layer of protective cartilage and soft tissue. It is this eggshell-like fragment that is avulsed that consists of cartilage, periosteum and retinaculum (1). The greatest challenge is the early diagnosis of these fractures, which are often missed or delayed because much of the fragment is unossified and can be missed on plain radiographs, these fractures are rare(4), and primary clinicians lack knowledge of them (5). At times the only indication of this fracture is a patella alta that is determined using the Insall-Salvati index. Neglect or delayed diagnosis is common and disastrous to the patient, who may go on to develop extensor lag and developmental disturbances of the patella (6). Our patient presented with a knee swelling after indirect trauma (pain while jumping for the ball in football); an initial diagnosis of patella tendon rupture had been made at a different facility. Misdiagnosis is common for the reasons already stated. Emphasis is on anatomical reduction and stabilization, which can be carried out using various methods. The aim of this paper is to highlight the importance of understanding the anatomy of an immature patella and of employing various teaching aids to make an early and accurate diagnosis. We with a back slab. Early diagnosis and surgical management are critical to favorable outcomes in these injuries.

Key words: Patella, Sleeve fracture, Pediatrics

Ann Afr Surg. 2019; 16(1):43-45

DOI: http://dx.doi.org/10.4314/aas.v16i1.11

\section{Conflicts of Interest: None}

Funding: None

(C) 2019 Author. This work is licensed under the Creative Commons Attribution 4.0 International License.

hope this will aid in reducing the number of neglected cases or those cases that have been misdiagnosed or delayed.

\section{Case Report}

A 12-year-old boy was seen at the Presbyterian Church of East Africa (PCEA) Kikuyu Hospital Orthopedic and Rehabilitation Center in February 2016 with complaints of pain and inability to extend the right knee. He incurred the injury after a fall while playing football, which he described as his knee 'giving way'. No previous incident was reported. On examination, swelling of the right knee was noticed with tenderness on palpation greater on the anterior aspect. A palpable gap on the inferior aspect of patella was recorded and a high riding patella noted. Straight leg test was negative. Radiographs showed a patella alta with an Insall-Salvati index $>1.4$ (Figure 1). A sleeve fracture of the right patella was seen with an intact patella tendon. The patient had previously been diagnosed with soft tissue injury to the knee at a peripheral facility and was managed conservatively. A second opinion before he came to our facility for further management stated a patella tendon tear. MRI to quantify the diagnosis could not be performed due to lack of funds. Hence a decision to operate was based on clinical and radiological findings.

The patella was accessed using a mid-line incision and the diagnosis was confirmed intra-operatively (Figures 2a and b). The sleeve fracture was repaired with a 2-0 mersilene ethicon fibre suture using mattress suturing technique, which we felt was a reasonably strong suture, and the construct was 


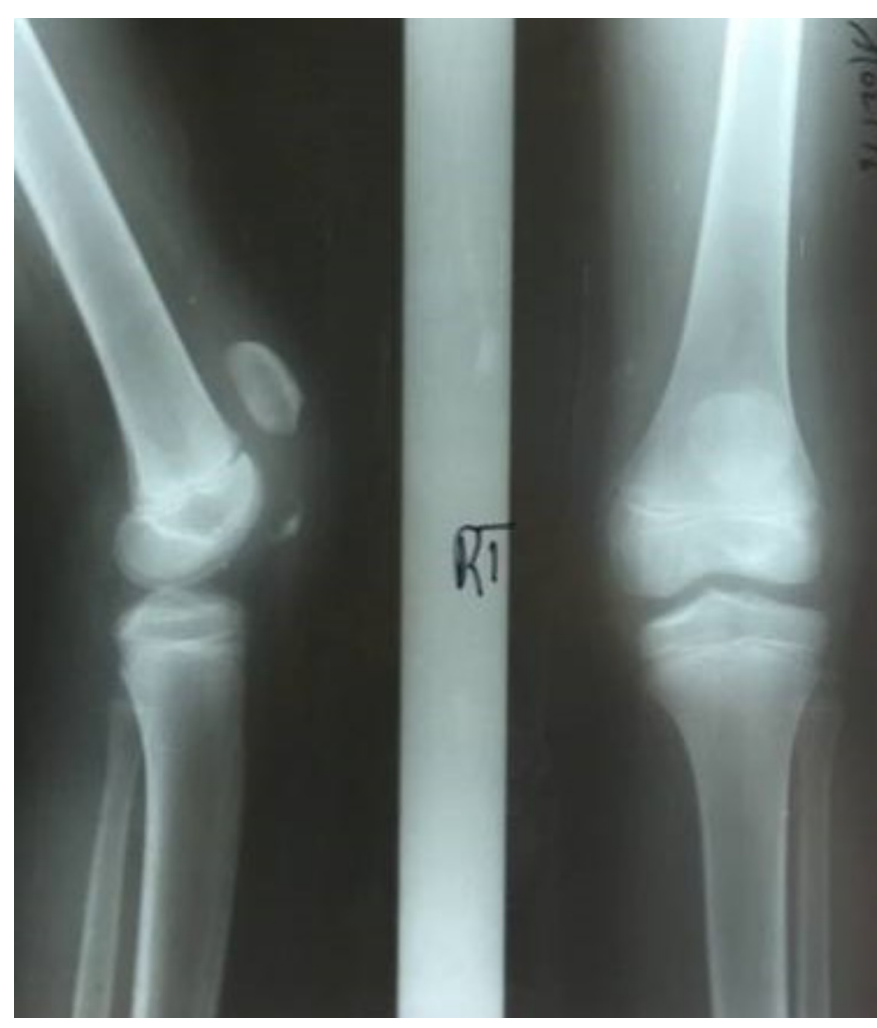

Figure 1: Radiographs of the right knee showing patella alta with an intact patella. Notice the small distal fragment on the lateral view that is easy to miss.

secured in a backslab. A cast was maintained for 6 weeks before removal (Figure 3). The patient was discharged on an above-knee plaster of Paris with nonweight bearing for 6 weeks. Unfortunately, he was lost to follow-up for reasons beyond our control. This denied us the opportunity to ascertain the final outcome of the patient.

\section{Discussion}

Patella fractures are rare in children and represent only $1 \%$ of fractures in the skeletally immature $(1,3)$. Sleeve fractures represent $57 \%$ of the patella fractures in this group

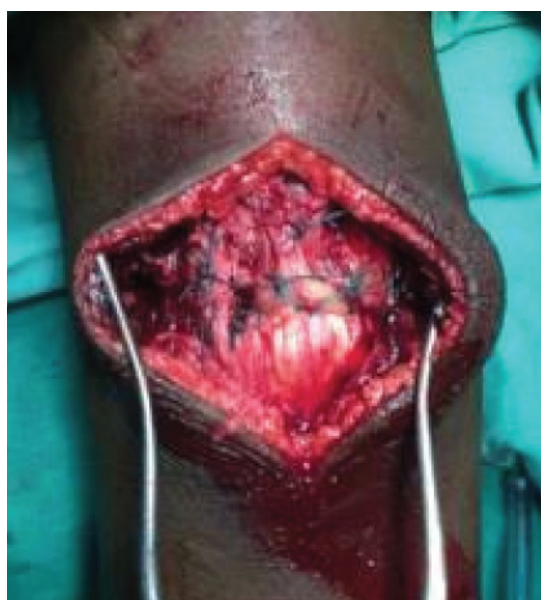

Figure 2a: Reduction of the sleeve fracture and held by sutures with repair of the retinaculum. Note the intact patella tendon.
(2). Sleeve fractures were first described by Houghton and Ackroyd in 1979 (5) and represent chondral or osteochondral avulsion at either pole of the patella, with the commonest being distal. The avulsion occurs along with articular cartilage, periosteum and retinaculum that is pulled off the main body of the structure (4). This is usually after forceful extension of the knee with the quadriceps contracting against resistance $(2,7)$.

The patella is the largest sesamoid bone in the body and an integral component in the extensor mechanism of the knee where it acts to increase the lever arm of the quadriceps. Patella ossification begins at 3-6 years of age and is usually complete by puberty. During ossification, the patella is surrounded by a layer of protective cartilage that makes the bulk of the patella. This feature and the fact that the patella is highly mobile in this group together and is less subjected to impact and tension forces during quadriceps contraction make patella fractures quite rare $(2,8)$. Hence, sleeve fractures are difficult to detect because much of the fragment is unossified and can be missed on plain radiographs, they are rare (4) and primary clinicians lack knowledge about them (5). They can be misinterpreted as a small bone avulsion of the pole of patella or they may be unrecognized if there is no ossified bone in the cartilaginous sleeve $(5,9)$. Anatomical variants such as a bipartite patella, nail patella syndrome and congenital distal pole fragmentation of patella may also be confused for patella sleeve fractures.

The greatest challenge here is the initial diagnosis, which is vital to a good outcome in the patient. Understanding of the anatomy of the immature patella is vital and a high index of suspicion is required in children with knee swelling without history of direct trauma, especially with a history of taking part in activities such as sports. Usually, patients will present with a knee swelling and unable to perform the straight leg raise with a palpable gap if the swelling is not marked. In our case, the patient had initially been managed conservatively, courtesy of misdiagnoses at two centers. We attribute this to lack of awareness of the condition and the subtle features on radiographs.

Misdiagnosis or delayed management of this condition can result in motion resistance in extension and developmental disturbances of the patella, leading to a double patella or a mega patella or even avascular necrosis (6). At times the only feature seen in a plain radiograph is a patella alta. Usually the patella length to patella tendon length is roughly $1: 1$, with a discrepancy of $20 \%$ on either side. 
The Insall-Salvati index is used to determine whether there is a patella alta or patella baja. The normal values are between 0.8 and 1.2. In some situations, a congenital patella alta may exist due to an abnormally long patella tendon, hence clinical history is important at all times. In this case, an initial diagnosis of patella tendon rupture was made in the outpatient clinic based on the patella alta seen on X-ray. We proceeded to calculate the InsallSalvati ratio to strengthen the diagnosis. MRI would have been helpful to confirm the diagnosis (2), but we couldn't perform it due to lack of funds.

Grogan et al. described four patterns of avulsion (sleeve)

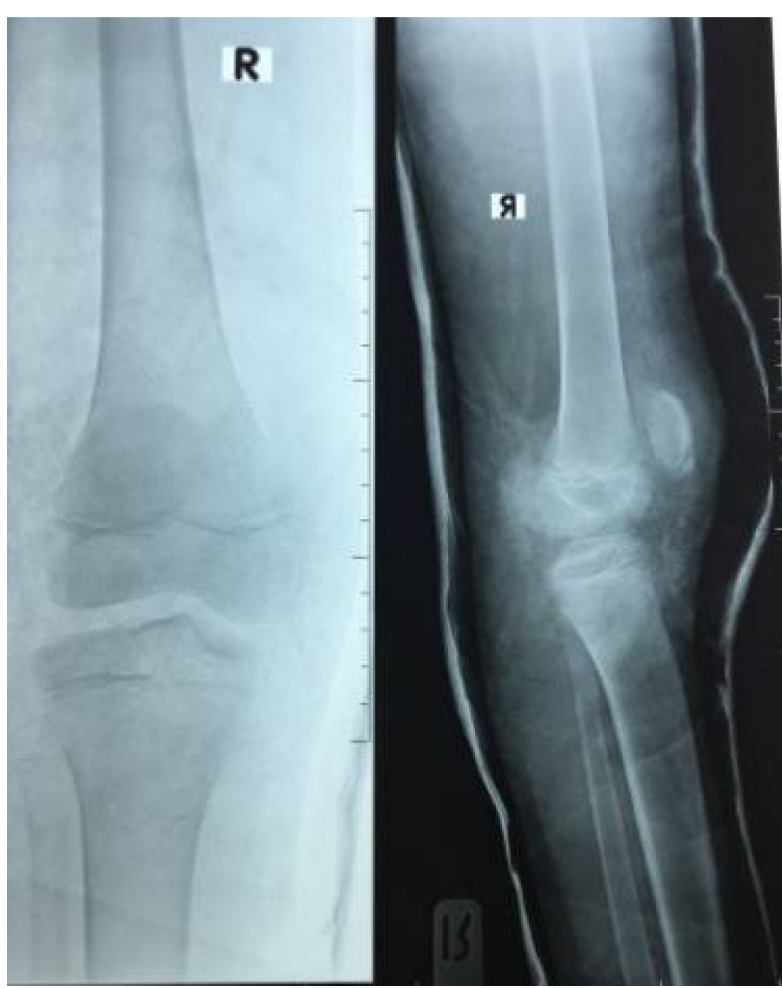

Figure 3: Postoperative radiograph showing reduced sleeve fracture together with a decrease in the Insall-Salvati index. fractures: superior, which is

the least common; inferior, which usually is caused by an acute injury; medial, which accompanies an acute lateral dislocation of the patella; and lateral, which is a chronic stress lesion resulting from repetitive tensile pull from the vastus lateralis muscle $(2,10)$. Our patient presented with an inferior avulsion, as shown by the intra-operative findings, which is consistent with the commonest type of avulsion. Conservative management can be advocated if there is minimal displacement. However, we recommend operative management with anatomical repositioning and a stable fixation. Again, emphasis is on early diagnosis and reducing the fracture to attain a favorable outcome for the patient (Figure 3). Fixation can be achieved with figure of eight sutures, anchors, transosseous sutures or even tension band wiring (11). We opted for repair with sutures as described earlier, which was the surgeon's preference, and immobilization for 6 weeks. Recognized complications of the surgery include infection, limited knee flexion, ectopic bone formation or even ischaemic necrosis of patella (2). We discharged the patient with the aim of a follow-up where we would look out for these complications. Unfortunately, he was lost to follow-up for reasons beyond our control. It is important to note the differential diagnoses that may cross the surgeon's mind during initial diagnosis. A congenitally long patella tendon may give a patella alta and confuse the diagnosis. Children with cerebral palsy may have a high-riding patella because of spastic tendons. Likewise, Osgood Schlatters disease and Sinding Larsen Johansen syndrome may mimic symptoms close to a sleeve fracture. A quadriceps tendon rupture and a true patella fracture should also be considered.

In conclusion, sleeve fractures are rare in children and $\mathrm{x}$-ray features are subtle, increasing the likelihood of delaying or missing a diagnosis, which may be disastrous to the patient. This paper highlights the need to understand the anatomy of an immature patella and to employ various teaching aids to make an early and accurate diagnosis. Management in most cases involves anatomic repositioning and stable fixation.

\section{References}

1. H Schmal PS. Fractures of patella in children and adolescents. Acta Orthop Belg. 2010; 76:644-50.

2. Shu-Yuan Lin, Wei-Chun Lin J-WW. Inferior sleeve fractures of patella: A case report. J Chinese Med Assoc. 2011; 74:101.

3. L Dai WZ. Fractures of patella in children. Knee Surg Sport Surg Traumatol Arthrosc. 1999; 7(4):243-5.

4. Guy S, Marciniak JN, Tulwa AC. Bilateral sleeve fractures of the inferior poles of the patella in a healthy child : A case report and review of literature. Adv Orthop. 2011;

5. G Houghton CA. Sleeve fractures of the patella in children : A report of three cases. J Bone Jt Surg [Br]. 1979; 61:165-8.

6. Labronici P, Fernandes H, et al. Patella fractures in children (sleeve type). Acta Orthop Bras. 2005; 13(5).

7. H Al-Khateeb KW. Sleeve fracture of patella: An unusual mechanism of injury. J Trauma. 2008; 65(1):8-9.

8. O Kameyamu RO. Sleeve fractures of patella in children: A review of two cases. J Kansai Med Univ. 1991; 43.

9. GX Gao, A Mahadev EL. Sleeve fractures of the patella in children. J Orthop Surg. 2008; 16(1):43-6.

10. Grogan D, Carey T, D Leffers JO. Avulsion fractures of the patella. J Pediat Orthop. 1990; 10(6):721-30.

11. T Van Isacker HDB. Sleeve fractures of the upper pole of the patella: A case report. Acta Orthop Belg. 2007; 73:114-7. 